DOI: $10.15593 / 2224-9982 / 2017.49 .07$

УДК 629.735.03-71:629.735.018.03

\author{
М.В. Пивоварова ${ }^{1,2}$, В.А. Бессчетнов ${ }^{2}$, А.В. Артемов ${ }^{2}$, В.М. Лимонова ${ }^{2}$ \\ ${ }^{1}$ Пермский национальный исследовательский политехнический университет, Пермь, Россия \\ ${ }^{2}$ АО «ОДК-Авиадвигатель», Пермь, Россия
}

\author{
ВЕРИФИКАЦИЯ РАСЧЕТА РАСПРЕДЕЛЕНИЯ ДИНАМИЧЕСКИХ \\ НАПРЯЖЕНИЙ И РАСЧЕТНОГО МЕТОДА РАЗРАБОТКИ СХЕМЫ \\ ПРЕПАРИРОВАНИЯ ДЛЯ РАБОЧЕЙ ЛОПАТКИ КОМПРЕССОРА
}

\begin{abstract}
В работе представлены результаты верификации расчета распределения динамических напряжений для рабочей лопатки. Верификация выполнена путем сравнения расчетных и экспериментальных данных. В работе также предложен расчетный метод разработки схемы препарирования для рабочей лопатки компрессора ГТД и выполнена его верификация. Расчетный метод разработки схемы препарирования основан на расчете собственных форм лопатки компрессора при комнатной температуре. Расчет собственных форм проведен в соответствии с лабораторными исследованиями на вибростенде. Для анализа качества схем препарирования было введено понятие коэффрициента чувствительности тензорезистора к фрорме колебаний. Для определения оптимального размещения тензорезисторов выделены три диапазона значений чувствительности по его применимости. Для достоверности выходных данных введен контроль напряжений при испытаниях по конкретной форме. Получены экспериментальные данные распределения динамических напряжений в лопатке компрессора. При сравнительном анализе выделено несколько групп по месту расположения тензорезисторов. Рассмотрены отклонения расчетных и экспериментальных эпюр вибронапряжений. Для каждого тензорезистора определено отклонение расчета от эксперимента. Определены среднее и максимальное отклонения распределения напряжений в тензорезисторах. Приведены результаты расчетного метода разработки схемы препарирования. Для лопатки компрессора определен диапазон применимости расчета собственных частот и расчета распределения динамических напряжений.
\end{abstract}

Ключевые слова: тензометрирование, коэффициент чувствительности тензорезистора к форме колебаний, вибронапряжения, эпюры распределения напряжений.

\author{
M.V. Pivovarova ${ }^{1,2}$, V.A. Besschetnov ${ }^{2}$, A.V. Artemov ${ }^{2}$, V.M. Limonova ${ }^{2}$ \\ ${ }^{1}$ Perm National Research Politechnic University, Perm, Russian Federation \\ ${ }^{2}$ ODK-Aviadvigatel JSC, Perm, Russian Federation
}

\title{
VERIFICATION OF THE ESTIMATION OF DYNAMIC STRESS DISTRIBUTION AND THE ESTIMATED METHOD OF PREPARATION DESIGN DEVELOPMENT FOR THE COMPRESSOR BLADE
}

\begin{abstract}
Verification results of the estimation of dynamic stress verification distribution for the blade are submitted. Verification is executed by comparison of estimation and experimental data. In the study, estimated method of preparation design development for the compressor blade of gas turbine engine also is offered and its verification is executed. The estimation method of preparation design development is based on estimation of compressor blade natural modes at room temperature. Estimation of natural frequencies is carried out according to laboratory researches on vibration table. For the analysis of quality of preparation design the concept of the strain gauge mode shape sensitivity factor is introduced. For definition of optimum of strain gauge placement, the three ranges of sensitivity values are allocated by its applicability. For reliability of the output data, the control of stress under tests under the concrete form is entered. Experimental data of dynamic stress distribution in compressor blade are obtained. At the comparative analysis some groups on strain gauge placement are allocated. The deviations of estimation dynamic stresses diagram from experimental data are considered. For every strain gauge, the deviation of estimation from experiment is determined. The average and maximal deviation of dynamic stresses distribution in strain gauge is determined. Results of estimation method of preparation design development are showed. The range of applicability of the estimation of natural frequencies and dynamic stress distribution is defined for compressor blades.
\end{abstract}

Keywords: strain gauge, strain gauge mode shape sensitivity factor, dynamic stresses, stresses distribution profile. 


\section{Введение}

Генеральным направлением развития газотурбинных двигателей является повышение термодинамических параметров рабочего цикла. Одним из средств обеспечения высоких показателей параметров цикла стало создание лопатки компрессора без бандажной полки.

Отсутствие антивибрационной полки позволяет улучшить аэродинамические характеристики при относительно малой массе лопатки, но проблема снижения уровня динамических напряжений для такой лопатки компрессора является весьма актуальной.

Для успешного проведения испытаний проектируемого газотурбинного двигателя необходим контроль вибрационного состояния деталей двигателя. Основным методом экспериментального контроля и оценки динамических напряжений в деталях ГТД является тензометрирование. Важной задачей при планировании тензометрирования становится разработка схем препарирования деталей. Схемы препарирования деталей могут быть разработаны на основе предварительных экспериментальных исследований в лабораторных условиях $[1,2]$ или на основе расчетного определения вибрационных характеристик и распределения динамических напряжений в лопатках [3, 4]. Расчеты собственных частот и форм колебаний проводятся с помощью конечно-элементного пакета моделирования. На основании этих расчетов разработаны методы определения оптимального размещения тензорезисторов, включающие в себя расчетную оценку коэффициента чувствительности тензорезистора к формам колебаний (ЧТФК).

Для повышения достоверности расчетов вибрационных характеристик, распределении я напряжений и метода разработки схем препарирования на основе расчетов в данной работе проведена верификация расчетов путем сравнения расчетов с экспериментальными исследованиями лопатки компрессора на вибростенде.

\section{Объект исследования}

Объектом исследования является рабочая лопатка, которая представляет собой лопасть рабочего колеса турбомашины. Лопатка состоит из пера, ножки и хвостовика замкового соединения. Ножка лопатки спрофилирована как продолжение пера с утолщением, с плавным переходом к хвостовику. Рабочая лопатка компрессора изготовлена из титана.

\section{Расчет вибрационных характеристик и распределения напряжений}

Расчет собственных форм колебаний проведен для условий испытаний на вибростенде, в качестве граничных условий берется запрет перемещений во всех направлениях по контактным граням хвостовика. В расчете задана температура лопатки $20^{\circ} \mathrm{C}$, соответствующая условиям лабораторного исследования на вибростенде ВЭДС-1500.

Расчет собственных форм проведен с использованием пакета конечно-элементного анализа конструкций ANSYS 14.0 [5-10]. При построении конечно-элементной модели использованы 20-узловые элементы SOLID186. Конечно-элементная модель состоит из 443454 узлов и 115432 элементов.

\section{Расчет коэффициентов чувствительности форм колебаний на лопатках компрессора при расчетном моделировании испытаний на вибростенде}

Коэффициент ЧТФК - это отношение величины измеряемого вибронапряжения по тензорезистору к максимальному значению вибронапряжения в детали. Коэффициент ЧТФК, получаемый по результатам расчета, запишется так: 


$$
K_{T}^{\text {pacч }}=\frac{\left[\sigma_{v}^{m / p}\right]^{\text {pacч }}}{\left[\sigma_{v}^{\text {max }}\right]^{\text {pacч }}},
$$

где $\left[\sigma_{v}^{m / p}\right]^{\text {расч }}-$ расчетное значение вибронапряжения по тензорезистору с учетом осреднения по базе тензорезистора; $\left[\sigma_{v}^{\max }\right]^{\text {расч }}-$ расчетное максимальное значение вибронапряжения в детали.

Выделим три диапазона для значений коэффициента $K_{T}^{\text {pacч}}$ :

1) приемлемые по величине значения - от 0,350 и выше (допустимой величиной коэффициента пересчета является $k=\left[\sigma_{v}^{\text {max }}\right]^{\text {расч }} /\left[\sigma_{v}^{m / p}\right]^{\text {pacч }} \leq 3$. Это соответствует значению $K_{T}^{\text {pacч }} \geq 0,35$, поскольку коэффициент ЧТФК - это величина обратная коэффициенту пересчета);

2) нежелательно приемлемые значения (использовать только при отсутствии приемлемых значений) - от 0,150 до 0,349 ;

3) неприемлемые значения - ниже 0,149 .

В зависимости от задач исследования можно ввести различные диапазоны приемлемости значений. В данном случае стоит задача «покрыть» как можно большее количество форм, поэтому введенного диапазона будет достаточно.

Контроль напряжений при испытаниях по конкретной форме будем считать возможным, если по этой форме:

- один и более тензорезисторов показывают приемлемые значения коэффициента ЧТФК;

- два или более тензорезисторов показывают «нежелательно приемлемые» значения коэффициента ЧТФК, при этом необходимо сравнивать показания двух и более тензорезисторов.

Для расчетного определения коэффициентов ЧТФК в тензорезисторах на детали сначала проводится расчет вибрационных характеристик - определяются значения собственных частот колебаний и распределение напряжений по собственным формам. После обработки результатов расчета вычисляются коэффициенты ЧТФК по формуле (1).

Тензорезистор измеряет деформации в одном направлении, а именно вдоль оси тензорезистора. Направление, или ориентация приклейки тензорезистора при препарировании лопатки, как правило, выбирается радиальным (вдоль кромки) или горизонтальным (вдоль хорды). Тензорезистор также может быть приклеен к лопатке под некоторым заданным углом к выбранному направлению. Деформация определяется в зоне, ограниченной размерами тензорезистора, в ней же проходит осреднение деформации.

Для определения коэффициента ЧТФК необходимо знать положение и вектор ориентации тензорезистора на лопатке, а также размеры тензорезистора.

В случае, если коэффициент ЧТФК принимает значение равное единице, тензорезистор находится в зоне максимальных напряжений. В том случае, если в процессе препарирования практически невозможно разместить тензорезистор в зоне максимальных напряжений, возможно разместить тензорезистор вблизи зоны максимальных напряжений. Соответственно, как правило, коэффициент ЧТФК принимает значение ниже единицы.

\section{Экспериментальные исследования вибрационных характеристик рабочей лопатки компрессора}

Экспериментальные исследования вибрационных характеристик рабочей лопатки компрессора в количестве двух штук с условными номерами 1 и 2 проведены при возбуждении на вибростенде в диапазоне частот $f=0 . .900$ Гц. По результатам экспериментальных исследований рабочих лопаток компрессора определены собственные частоты и распределения вибронапряжений по профилю лопатки [11-15]. 
При экспериментальном исследовании использовано две схемы препарирования лопатки. Условная схема препарирования приведена на рис. 1.

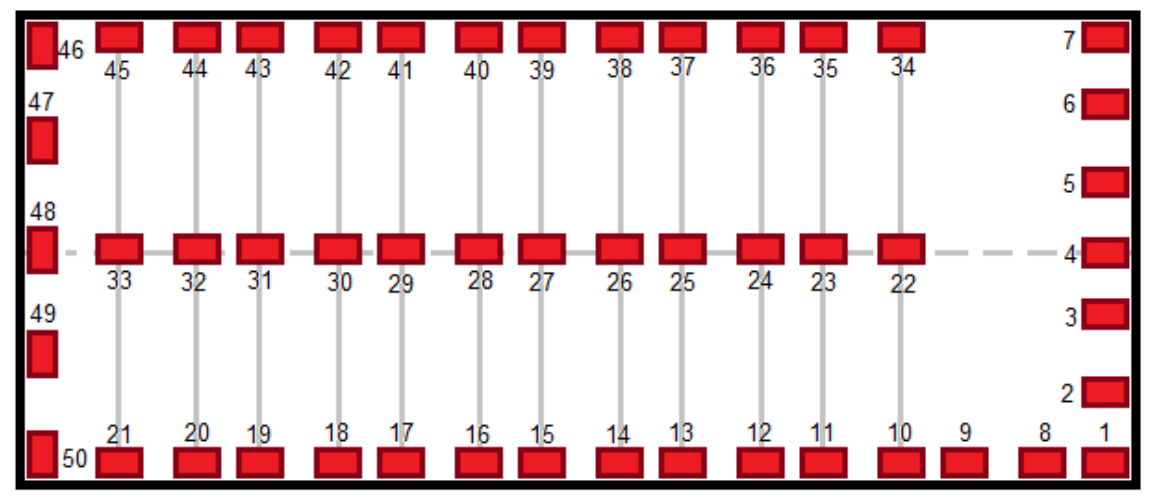

Рис. 1. Условная схема препарирования

Первый вариант схемы препарирования состоит из 50 тензорезисторов. Тензорезисторы № 1-7, 22-33, 46-50 расположены со стороны спинки, тензорезисторы № 8-21, 34-45 расположены со стороны корыта.

Второй вариант схемы препарирования состоит из 60 тензорезисторов. Схема препарирования для лопаток была доработана по сравнению с первым вариантом с помощью добавления новых тензорезисторов. Тензорезисторы № 1-7, 22-33, 46-59 расположены со стороны спинки, тензорезисторы № 8-21, 34-45, 40' расположены со стороны корыта. 6,35 мм.

Для первой схемы применялись тензорезисторы базой 5 мм, для второй схемы - базой

В представленных схемах препарирования тензорезисторы расположены в радиальном направлении (№ 1-7, 22-33), в направлении вдоль периферии (№ 46-50) и вдоль кромок лопатки (№ 8-21, 34-45).

\section{Сравнение результатов расчета с экспериментальными исследованиями}

По результатам экспериментальных исследований рабочих лопаток компрессора определены:

- собственные частоты (в относительных величинах по отношению к первой изгибной форме), представленные в табл. 1;

Таблица 1

Сравнительный анализ собственных частот колебаний лопаток по результатам расчета и лабораторного исследования

\begin{tabular}{|c|l|c|c|c|c|c|}
\hline \multirow{2}{*}{$\begin{array}{c}\text { Номер } \\
\text { формы }\end{array}$} & \multirow{2}{*}{$\begin{array}{c}\text { Форма } \\
\text { колебания }\end{array}$} & \multicolumn{4}{|c|}{ Частота, у.е. } & \multirow{2}{*}{$\begin{array}{c}\text { По результатам экспериментального } \\
\text { исследования }\end{array}$} \\
\cline { 3 - 5 } & & По результатам & $\begin{array}{c}\text { Отконение, } \\
\text { расчета }\end{array}$ & \\
\hline$f_{1}$ & 1-я изгибная & 1,01 & 0,99 & 1,0 & 1,0 & 0,4 \\
\hline$f_{2}$ & 2-я изгибная & 2,82 & 2,82 & 2,8 & 2,87 & 2 \\
\hline$f_{3}$ & 1-я крутильная & 4,58 & 4,61 & 4,60 & 4,83 & 5 \\
\hline$f_{4}$ & 3-я изгибная & 7,26 & 7,2 & 7,23 & 7,63 & 6 \\
\hline$f_{5}$ & 2-я крутильная & 10,17 & - & 10,17 & 10,86 & 6,8 \\
\hline
\end{tabular}


- распределения вибронапряжений в относительных величинах (по отношению к максимально замеренным напряжениям) или эпюры вибронапряжений по проявившимся собственным формам.

По результатам расчета определены собственные частоты (см. табл. 1) и распределения вибронапряжений в лопатках по собственным формам.

Анализ первого и второго вариантов схем препарирования лопаток компрессора показал практически одинаковые результаты по распределению коэффициента ЧТФК. Различия состоят в разном количестве тензорезисторов на первом и втором вариантах схем препарирования по периферии и выходной кромке со стороны корыта.

В табл. 2 по собственным формам лопатки приведен пример расположения зон с максимальными напряжениями и максимальные значения коэффициентов ЧТФК в тензорезисторах по рассматриваемым схемам препарирования. Из табл. 3 видно, что максимальные значения коэффициента ЧТФК в тензорезисторах от 0,5 до 0,9 .

Таблица 2

Пример расположения зон максимальных напряжений в лопатке компрессора

\begin{tabular}{|c|l|c|c|l|}
\hline \multirow{2}{*}{$\begin{array}{c}\text { Номер } \\
\text { формы }\end{array}$} & \multirow{2}{*}{$\begin{array}{c}\text { Описание зоны с максимальными } \\
\text { напряжениями, полученными } \\
\text { по расчету }\end{array}$} & \multicolumn{3}{|c|}{ Тензорезистор с максимальным значением коэффициента } \\
\cline { 3 - 4 } & \multicolumn{2}{|c|}{$\begin{array}{c}\text { Номер } \\
\mathrm{T} / \mathrm{p}\end{array}$} & $\begin{array}{c}\text { Значение коэфф. } \\
\text { ЧТФК }\end{array}$ & Описание зоны размещения т/p \\
\hline$f_{1}$ & На ножке со стороны корыта & 8 & 0,802 & Рядом с ножкой на корыте со \\
\cline { 3 - 4 }$f_{2}$ & на входной кромке & 8 & 0,800 & стороны входной кромки \\
\hline$f_{3}$ & $\begin{array}{l}\text { На ножке со стороны корыта } \\
\text { на выходной кромке }\end{array}$ & 40 & 0,674 & $\begin{array}{l}\text { На корыте со стороны выход- } \\
\text { ной кромки посередине }\end{array}$ \\
\hline$f_{4}$ & $\begin{array}{l}\text { На ножке со стороны корыта } \\
\text { на входной кромке }\end{array}$ & 8 & 0,799 & $\begin{array}{l}\text { Рядом с ножкой на корыте со } \\
\text { стороны входной кромки }\end{array}$ \\
\hline$f_{5}$ & $\begin{array}{l}\text { На корыте со стороны входной } \\
\text { кромки вверху }\end{array}$ & 20 & 0,914 & $\begin{array}{l}\text { На корыте со стороны входной } \\
\text { кромки вверху }\end{array}$ \\
\hline
\end{tabular}

В табл. 1 представлено сравнение собственных частот, полученных расчетным путем, с результатами эксперимента. Отклонение по собственным частотам результатов эксперимента от расчета незначительно. С ростом формы и собственной частоты отклонение растет, максимальное отклонение по второй крутильной форме 6,8\%.

По результатам расчета и эксперимента сравниваются собственные частоты лопатки компрессора.

Сравниваются тензорезисторы с максимальными напряжениями и максимальным коэффициентом ЧТФК, полученными по результату эксперимента и расчета.

Для сравнения распределения вибронапряжений рассматривается отклонение расчетных и экспериментальных эпюр вибронапряжений. Построение эпюр распределения напряжений по результатам эксперимента проводится в следующем порядке:

- определяется тензорезистор с максимальным значением напряжения;

- в каждом тензорезисторе рассматривается отношение значения напряжения к максимальному значению напряжения: $K_{T}=\frac{\sigma}{\sigma_{\max }}$. В тензорезисторе с максимальным значением напряжения данное соотношение равно 1,0 ;

- строятся эпюры вибронапряжений в лопатке для групп тензорезисторов, например в зависимости от расстояния от подошвы замка.

При расчете коэффициентов ЧТФК в тензорезисторе рассматривается соотношение по отношению к максимальному напряжению в лопатке. В зоне максимальных напряжений, как 
правило, разместить тензорезистор невозможно, поэтому для сравнения расчетных и экспериментальных эпюр распределения напряжений масштабируем расчетные значения коэффициента ЧТФК для каждого тензорезистора по максимальному абсолютному значению коэффициента ЧТФК:

$$
\left[K_{T}^{\text {pacч }}\right]^{\text {масш }}=\frac{K_{T}^{\text {pacч }}}{\max \left|K_{T}^{\text {pacч }}\right|},
$$

где $K_{T}^{\text {расч }}-$ коэффициент ЧТФК; $\max \left|K_{T}^{\text {расч}}\right|-$ максимальное абсолютное значение коэффициента ЧТФК из всех тензорезисторов.

Для сравнения расчетных и экспериментальных эпюр распределения напряжений рассмотрено отношение

$$
K_{1}=\frac{K_{T}^{\text {эксп }}}{\left[K_{T}^{\text {pacч }}\right]^{\text {масш }}} .
$$

Также оценивается отклонение эксперимента и расчета:

$$
\Delta=\frac{\left|K_{T}^{\text {эксп }}-\left[K_{T}^{\text {расч }}\right]^{\text {масш }}\right|}{\left|\left[K_{T}^{\text {расч }}\right]^{\text {масш }}\right|} \cdot 100 \% .
$$

При сравнительном анализе выделено несколько групп по месту расположения тензорезисторов: подошва по спинке, входная кромка по корыту, вдоль по спинке, выходная кромка по корыту и периферия по спинке. В каждой группе выделены подгруппы по диапазонам значений коэффициента ЧТФК: приемлемые, нежелательно приемлемые, неприемлемые (описано выше). Для каждого тензорезистора определено отклонение расчета от эксперимента. Для подгрупп определены среднее и максимальное отклонения.

Результаты сравнительного анализа по первым пяти формам на входной кромке по корыту, вдоль по спинке, по выходной кромке по корыту представлены на рис. 2.

В табл. 3 приведен пример отклонения эксперимента и расчета для тензорезисторов по приемлемым и по нежелательно приемлемым тензорезисторам.

По рекомендации М.Е. Колотникова и П.В. Макарова величину допустимого отклонения лабораторного исследования от расчета необходимо установить не более $25 \%$ [7]. Предполагается, что при отклонении больше $25 \%$ от измеряемого значения данные тензорезисторы неприменимы для оценки максимальных вибронапряжений.

Результаты сравнения для первой и второй форм колебаний лопатки показали, что для тензорезисторов:

- с приемлемыми значениями коэффициентов ЧТФК среднее отклонение расчета и эксперимента меньше $9 \%$, а максимальное отклонение меньше $25 \%$;

- с нежелательно приемлемыми значениями коэффициентов ЧТФК среднее отклонение расчета и эксперимента меньше $17 \%$, а максимальное отклонение меньше $40 \%$.

По третьей форме колебаний наблюдается увеличение отклонений расчетных и экспериментальных значений, а именно для тензорезисторов:

- с приемлемыми значениями коэффициентов ЧТФК среднее отклонение расчета и эксперимента составляет $17 \%$, а максимальное отклонение равно $37 \%$;

- с нежелательно приемлемыми значениями коэффициентов ЧТФК среднее отклонение расчета и эксперимента составляет $15 \%$, а максимальное отклонение равно $70 \%$. 


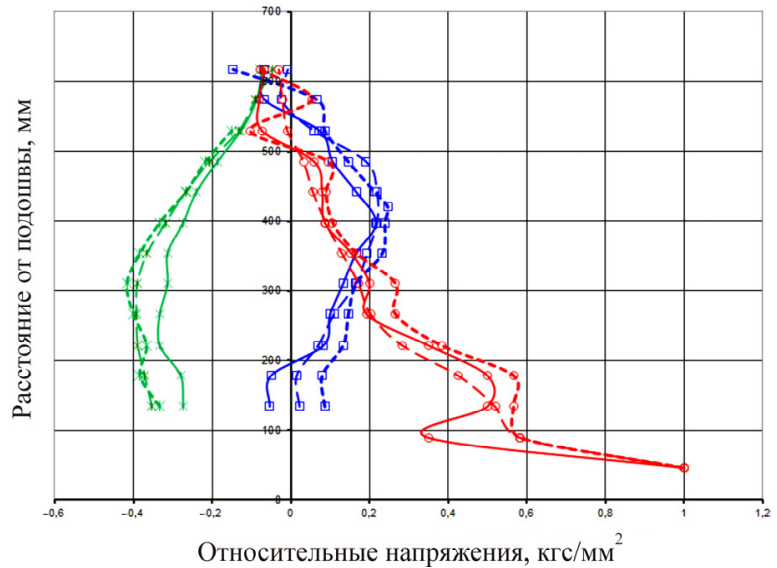

a

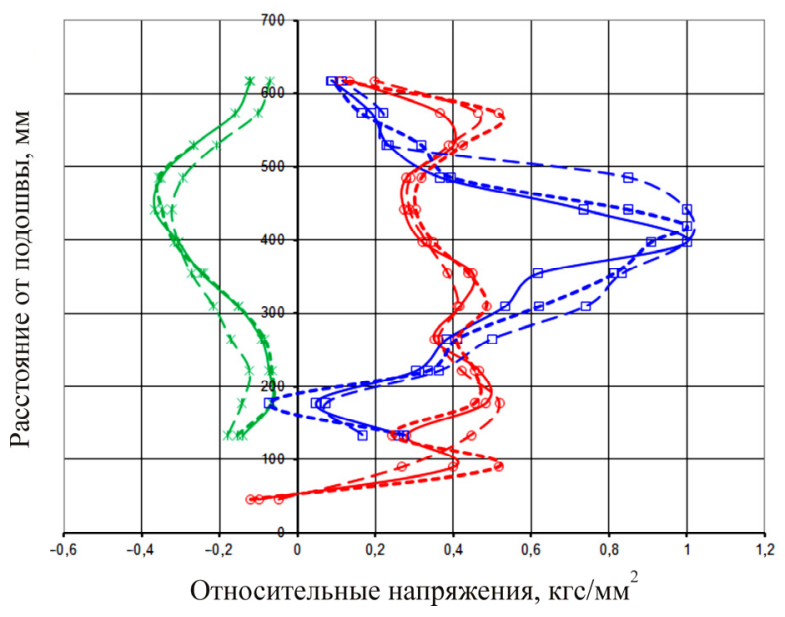

b

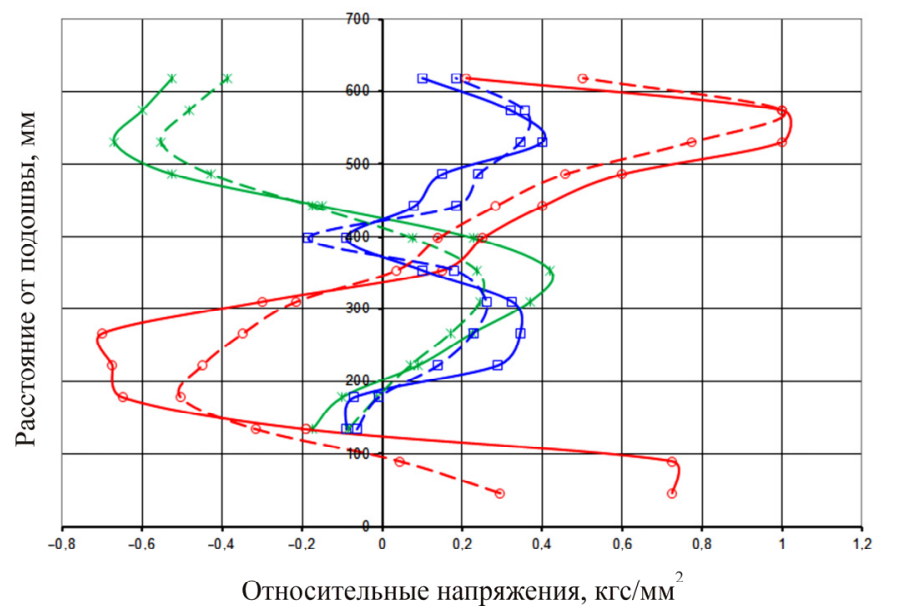

$\partial$

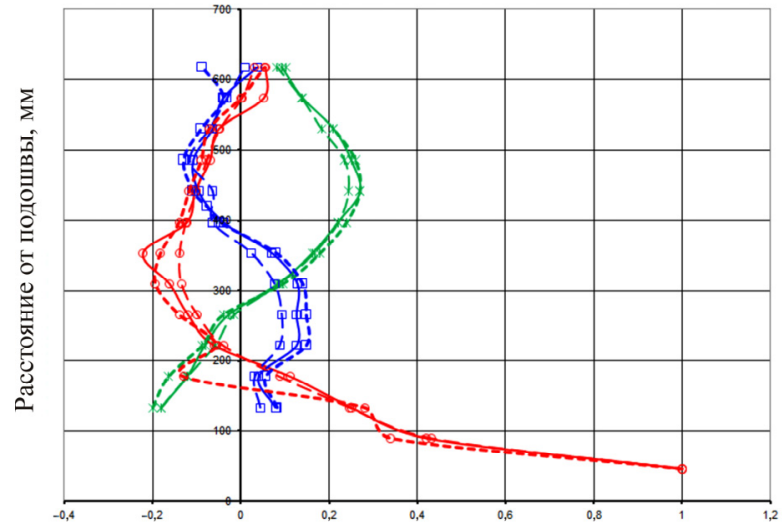

Относительные напряжения, кгс/мм²

$\sigma$

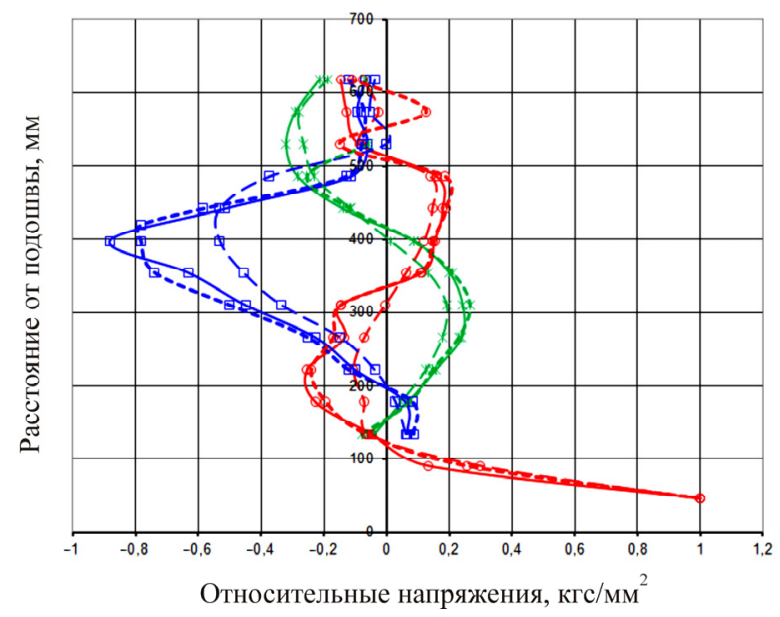

2

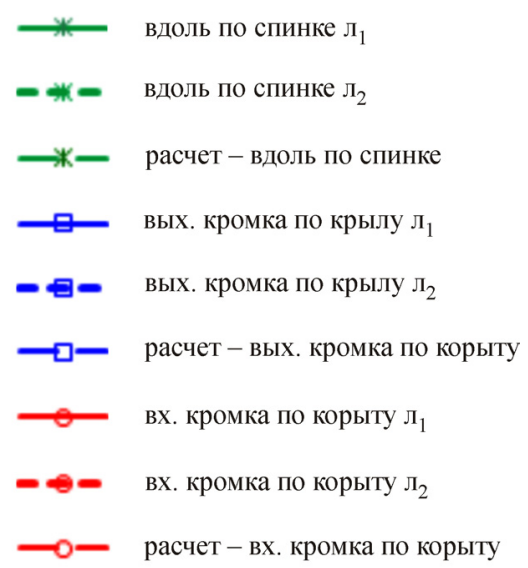

Рис. 2. Распределение вибронапряжений в лопатке компрессора при колебаниях лопатки с относительными частотами $f_{1}=1,0(a) ; f_{2}=2,87(\sigma) ; f_{3}=4,83($ ( ) $) ; f_{4}=7,63(2) ; f_{5}=10,86(\partial)$ 
Пример отклонения распределения напряжений в тензорезисторах

\begin{tabular}{|c|c|c|c|}
\hline \multirow{2}{*}{$\begin{array}{l}\text { Номер } \\
\text { формы }\end{array}$} & \multirow{2}{*}{ Форма колебания } & \multicolumn{2}{|c|}{ Отклонение, \% } \\
\hline & & Среднее & Максимальное \\
\hline \multirow{2}{*}{$f_{1}$} & \multirow{2}{*}{ 1-я изгибная } & $8,85 *$ & 24,5 \\
\hline & & $16,2 * *$ & 40,0 \\
\hline \multirow{2}{*}{$f_{2}$} & \multirow{2}{*}{ 2-я изгибная } & $\mathbf{0 , 0}$ & $\mathbf{0 , 0}$ \\
\hline & & 9,5 & 16,0 \\
\hline \multirow[b]{2}{*}{$f_{3}$} & \multirow{2}{*}{ 1-я крутильная } & 16,9 & 36,4 \\
\hline & & 14,3 & 70,4 \\
\hline \multirow{2}{*}{$f_{4}$} & \multirow{2}{*}{ 3-я изгибная } & 35,0 & 64,4 \\
\hline & & 41,3 & 84,8 \\
\hline \multirow{2}{*}{$f_{5}$} & \multirow{2}{*}{ 2-я крутильная } & 40,1 & 100,0 \\
\hline & & 61,6 & $>100$ \\
\hline
\end{tabular}

* - жирным - по приемлемым тензорезисторам; ** - курсивом - по нежелательно приемлемым тензорезисторам.

Для более высоких форм колебаний наблюдается повышение величин отклонений более чем на $30 \%$.

Для тензорезисторов с неприемлемыми значениями коэффициентов ЧТФК отклонения расчета и эксперимента могут быть больше $100 \%$.

\section{Заключение}

По результатам сравнения расчета и эксперимента можно сделать следующие выводы:

1. Проведена верификация расчета распределения динамических напряжений и расчетного метода разработки схемы препарирования для рабочей лопатки компрессора.

2. Расчетный метод определения распределения вибронапряжений и оценки коэффициентов чувствительности тензорезисторов к формам колебаний для лопатки компрессора применим для первых трех форм колебаний.

3. Для оценки вибронапряжений при тензометрировании рабочей лопатки компрессора можно использовать только тензорезисторы с приемлемым значением коэффициентов ЧТФК.

\section{Библиографический список}

1. Собственные частоты и формы колебаний полой лопатки вентилятора ГТД / А.А. Иноземцев, М.Ш. Нихамкин, Л.В. Воронов, Б.П. Болотов, И.Л. Гладкий, А.Ю. Головкин // Авиационная промышленность. -2010 . - № 3. - С. 8-11.

2. Методика экспериментального модального анализа лопаток и рабочих колес газотурбинных двигателей / А.А. Иноземцев, М.Ш. Нихамкин, Л.В. Воронов, А.Б. Сенкевич, А.Ю. Головкин, Б.П. Болотов // Тяжелое машиностроение. - 2010. - № 11. - С. 2-6.

3. Отработка расчетного метода снижения динамических напряжений в пустотелой лопатке вентилятора / В.А. Бессчетнов, М.В. Пивоварова, Л.В. Сараева, А.А. Стром // Сб. тез. НТКД-2014, Москва, 15-17 апреля 2014. - М., 2014. - С. 174-177.

4. Бикмеев А.Т., Иванов С.В., Перепелица С.И. Компьютерное моделирование процесса остывания полой лопатки ГТД после сверхпластической формовки // Вестник УГАТУ. - 2012. - Т. 16, № 7(52). C. $38-42$.

5. Иванов В.П. Колебание рабочих колес турбомашин. - М.: Машиностроение, 1983. - С. 205.

6. Биргер И.А., Шорр Б.Ф., Иоселевич Г.Б. Расчет на прочность деталей машин: справочник. - М.: Машиностроение, 1979. - 702 с.

7. Колотников М.Е., Макаров П.В. Один из подходов к оценке динамической напряженности лопаток вентилятора при тензометрировании // Вестник двигателестроения. - 2009. - № 3. - С. 176-180. 
8. Тимошенко С.П., Гудьер Дж. Теория упругости. - М.: Наука, 1975. - 576 с.

9. Основы технологии создания газотурбинных двигателей для магистральных самолетов / А.Г. Братухин, Ю.Е. Решетников, А.А. Иноземцев [и др.]. - М.: Авиатехинформ, 1999. - 553 с.

10. Пригоровский Н.И. Методы и средства определения полей деформаций и напряжений: справочник. - М.: Машиностроение, 1983. - 248 с.

11. Асатурян В.И. Теория планирования эксперимента: учеб. пособие для вузов. - М.: Радио и связь, 1983. - 248 с.

12. Аш Ж.И. Датчики измерительных систем. - М.: Мир, 1992. - 480 с.

13. Леонтьев В.Н., Сиротин С.А., Теверовский А.М. Испытания авиационных двигателей и их агрегатов. - М.: Машиностроение, 1976. - 212 с.

14. Ножницкий Ю.А., Куевда В.К., Мокроус М.Ф. Сертификация авиационных двигателей // ЦИАМ, 1980-2000. Кн. 1. Научный вклад в создание авиационных двигателей. - М.: Машиностроение, 2000. - C. 669-374.

15. Солохин Э.Л. Испытания воздушно-ракетных двигателей. - М.: Машиностроение, 1975. - 356 с.

\section{Reference}

1. Inozemtsev A.A., Nihamkin M.Sh., Voronov L.V., Bolotov B.P., Gladky I.L., Golovkin A.Yu. Sobstvennye chastoty i formy kolebaniy poloy lopatki ventilyatora GTD [Natural frequencies and mode shapes of the hollow fan blades of GTE]. Aviatsionnaya promyshlennost, 2010, no. 3, pp. 8-11.

2. Inozemtsev A.A., Nihamkin M.Sh., Voronov L.V., Sienkiewicz A.B., Golovkin A.Yu., Bolotov B.P. Metodika eksperimentalnogo modalnogo analiza lopatok i rabochikh koles gazoturbinnykh dvigateley [Methods of experimental modal analysis of blades and impellers turbine engines]. Tyazheloe mashinostroenie, 2010, № 11, pp. 2-6.

3. Besschetnov V.A., Pivovarova M.V., Sarajevo L.V., Strom A.A. Otrabotka raschetnogo metoda snizheniya dinamicheskikh napryazheniy $\mathrm{v}$ pustoteloy lopatke ventilyatora [Testing of calculation method reduce dynamic stresses in the hollow fan blades]. Sbornik tezisov NTKD-2014, Moscow, 15-17 April 2014, pp. 174-177.

4. Bikmeev A.T., Ivanov S.V., Perepelitsa S.I. Perepelitsa S.I. Kompyuternoe modelirovanie protsessa ostyvaniya poloy lopatki GTD posle sverkhplasticheskoy formovki [The computer simulation of the hollow blade cooling process after superplastic forming]. Vestnik Ufimskogo gosudarstvennogo aviatsionnogo tekhnicheskogo universiteta, 2012, vol. 16, no. 7 (52), pp. 38-42.

5. Ivanov V.P. Kolebanie rabochikh koles turbomashin [Swing turbomachinery impellers]. Moscow, Mashinostroenie, 1983, pp. 205.

6. Birger I.A., Shorr B.F., Ioselevich G.B. Raschet na prochnost detaley mashin: Spravochnik [Strength analysis of machine parts: Handbook]. Moscow, Mashinostroenie, 1979, 702 p.

7. Kolotnikov M.E., Makarov P.V. Odin iz podkhodov k otsenke dinamicheskoy napryazhennosti lopatok ventilyatora pri tenzometrirovanii [One approach to the assessment of the dynamic tension of the fan blades at the strain measurement]. Vestnik dvigatelestroeniya. 2009, no. 3, pp. 176-180.

8. Timoshenko S.P., Guder Dzh. Teoriya uprugosti [Theory of elasticity]. Moscow, Nauka, 1975, 576 p.

9. Bratukhin A.G., Reshetnikov Yu.E., Inozemtsev A.A. et al. Osnovy tekhnologii sozdaniya gazoturbinnykh dvigateley dlya magistralnykh samoletov [Bases of technology of creation of gas-turbine engines for the main planes]. Moscow, Aviatekhinform, 1999, 553 p.

10. Prigorovskiy N.I. Metody i sredstva opredeleniya poley deformatsiy i napryazheniy: Spravochnik [Methods and means of definition of fields of deformations and tension: Reference book]. Moscow, Mashinostroenie, 1983, $248 \mathrm{p}$.

11. Asaturyan V.I. Teoriya planirovaniya eksperimenta [The theory of planning of experiment]. Moscow, Radio i svyaz, 1983, 248 p.

12. Ash Zh.I. Datchiki izmeritelnykh system [Sensors and measuring systems]. Moscow, Mir, 1992, 480 p.

13. Leontev V.N., Sirotin S.A., Teverovskiy A.M. Ispytaniya aviatsionnykh dvigateley i ikh agregatov [Testing of aircraft engines and their components]. Moscow, Mashinostroenie, 1976, 212 p.

14. Nozhnitskiy Yu.A., Kuevda V.K., Mokrous M.F. Sertifikatsiya aviatsionnykh dvigateley [Certification of aircraft engines]. Moscow: Mashinostroenie, 2000.

15. Solokhin E.L. Ispytaniya vozdushno-raketnykh dvigateley [Test of the air-rocket engines]. Moscow, Mashinostroenie, 1975, $356 \mathrm{p}$. 


\section{Об авторах}

Пивоварова Мария Владимировна (Пермь, Россия) - аспирантка кафедры «Авиационные двигатели» ФГБОУ ВО ПНИПУ; инженер-конструктор-расчетчик 3-й категории АО «ОДК-Авиадвигатель» (614990, г. Пермь, Комсомольский пр., д. 93, e-mail: pivovarova-mv@avid.ru).

Бессчетнов Вадим Андреевич (Пермь, Россия) - начальник бригады перспективных методов анализа динамики и прочности АО «ОДК-Авиадвигатель» (614990, г. Пермь, Комсомольский пр., д. 93, e-mail: besschetnov-va@avid.ru).

Артемов Алексей Владимирович (Пермь, Россия) - инженер АО «ОДК-Авиадвигатель» (614990, г. Пермь, Комсомольский пр., 93).

Лимонова Валентина Михайловна (Пермь, Россия) - начальник бригады лопаток АО «ОДКАвиадвигатель» (614990, г. Пермь, Комсомольский пр., д. 93).

\section{About the authors}

Maria V. Pivovarova (Perm, Russian Federation) - Postgraduate Student, Department of Aviation Engines, Perm National Research Polytechnic University, Design Engineer, ODK-Aviadvigatel JSC (93, Komsomolsky av., Perm, 614990, Russian Federation, e-mail: pivovarova-mv@avid.ru).

Vadim A. Besschetnov (Perm, Russian Federation) - Head of the Team of Promising Methods for the Analysis of the Dynamics and Strength, ODK-Aviadvigatel JSC (93, Komsomolsky av., Perm, 614990, Russian Federation, e-mail: besschetnov-va@avid.ru).

Aleksey V. Artemov (Perm, Russian Federation) - Engineer, ODK-Aviadvigatel JSC (93, Komsomolsky av., Perm, 614990, Russian Federation).

Valentina M. Limonova (Perm, Russian Federation) - Head of the Team of the Blades, ODKAviadvigatel JSC (93, Komsomolsky av., Perm, 614990, Russian Federation).

Получено 28.02.2017 